\title{
First Direct Observation Of Enhanced Octupole Collectivity in ${ }^{146} \mathrm{Ba}$
}

B. Bucher ${ }^{*}{ }^{a b}$ S. Zhu, ${ }^{c}$ C. Y. Wu, ${ }^{a}$ R. V. F. Janssens, ${ }^{c}$ D. Cline, ${ }^{d}$ A. B. Hayes, ${ }^{d}$

A. D. Ayangeakaa, ${ }^{c}$ M. Q. Buckner, ${ }^{a}$ C. M. Campbell, ${ }^{e}$ M. P. Carpenter, ${ }^{c}$ J. A. Clark, ${ }^{c}$ H. L. Crawford, ${ }^{e}$ H. M. David, ${ }^{c}$ C. Dickerson, ${ }^{c}$ J. Harker, ${ }^{c}$ C. R. Hoffman, ${ }^{c}$ B. P. Kay, ${ }^{c}$ F. G. Kondev, ${ }^{c}$ T. Lauritsen, ${ }^{c}$ A. O. Macchiavelli, ${ }^{e}$ R. C. Pardo, ${ }^{c}$ G. Savard, ${ }^{c}$ D. Seweryniak ${ }^{c}$ and R. Vondrasek ${ }^{c}$

${ }^{a}$ Lawrence Livermore National Laboratory Livermore, California 94550, USA

${ }^{b}$ Idaho National Laboratory Idaho Falls, Idaho 83415, USA

${ }^{c}$ Argonne National Laboratory Argonne, Illinois 60439, USA

${ }^{d}$ University of Rochester Rochester, New York 14627, USA

e Lawrence Berkeley National Laboratory

Berkeley, California 94720, USA

E-mail: brian.buchereinl.gov

The octupole strength present in the neutron-rich, radiocative nucleus ${ }^{146} \mathrm{Ba}$ has been experimentally determined for the first time using Coulomb excitation. To achieve this, $A=146$ fission fragments from CARIBU were post-accelerated by the Argonne Tandem Linac Accelerator System (ATLAS) and impinged on a thin ${ }^{208} \mathrm{~Pb}$ target. Using the GRETINA $\gamma$-ray spectrometer and the $\mathrm{CHICO} 2$ heavy-ion counter, the reduced transition probability $B\left(E 3 ; 3^{-} \rightarrow 0^{+}\right)$was determined as $48\left({ }_{-29}^{+21}\right)$ W.u. The new result provides further experimental evidence for the presence of a region of octupole deformation surrounding the neutron-rich barium isotopes.

PACS: 27.60.+j, 25.70.De, 29.38.Gj, 23.20.Js, 23.20.-g, 21.10.KY

The 26th International Nuclear Physics Conference

11-16 September, 2016

Adelaide, Australia

* Speaker. 


\section{Introduction}

The first experimental evidence indicating that the atomic nuclei of certain isotopes exhibit octupole-deformed shapes was provided several decades ago through the observations of low-lying $1^{-}$states populated in $\alpha$ decay [1]. Since then, there has been a wealth of experimental and theoretical investigations attempting to understand and characterize the importance of octupole correlations in nuclei (see Ref. [2] and references therein). Indeed, there is now ample evidence to suggest that the most octupole-deformed nuclei will generally display a low-lying negative-parity band of states that interleaves with the ground-state $0^{+}$band with relatively fast $E 1$ transitions linking the two. The strongest octupole correlations have been observed in isotopes having certain combinations of protons and neutrons, namely $Z, N \sim 34,56,88,134$ [3], which is where valence nucleons occupy pairs of single-particle orbitals near the Fermi surface that differ in orbital $(\ell)$ and total $(j)$ angular momenta by 3 units of $\hbar$ [2]. However, the magnitude of octupole strength present in what is expected to be the two most octupole-enhanced regions, has only recently been quantified in the neutron-deficient radium isotopes [4, 5] and the neutron-rich barium isotopes [6] providing the only direct experimental evidence for octupole-deformed nuclear shapes. Such kind of measurements are difficult since they involve Coulomb excitation of radioactive beams or targets. In particular, the radioactive barium isotopes relevant for these measurements are especially difficult to measure since the lifetimes are on the order of seconds only. Those experiments have only been made possible within the past few years through the development of re-accelerated radioactive beam facilities such as CARIBU [7, 8] at Argonne National Lab or REX-ISOLDE [9] at CERN. The following report highlights the newest of these studies where octupole measurements have been extended to the neutron-rich isotope ${ }^{146} \mathrm{Ba}$ with $N=90$.

\section{Experiment}

As stated above, in order to investigate the octupole strength in ${ }^{146} \mathrm{Ba}$, a Coulomb excitation experiment was performed similar to the one done for ${ }^{144} \mathrm{Ba}$ [6]. The experimental setup was identical utilizing the CHICO2 heavy ion counter [10] and the GRETINA $\gamma$-ray tracking array [11], while the radioactive ${ }^{146} \mathrm{Ba}\left(T_{1 / 2}=2.2 \mathrm{sec}\right.$. [12] $)$ beam was produced by the CARIBU ${ }^{252} \mathrm{Cf}$ fission source $[7,8]$ and accelerated through the Argonne Tandem Linac Accelerator System (ATLAS). In addition to the beam of ${ }^{146} \mathrm{Ba}$ ions, CARIBU also produced the isobaric contaminants ${ }^{146} \mathrm{La}$ and ${ }^{146} \mathrm{Ce}$ which could not be separated. The $A=146$ beam was injected into an electron cyclotron resonance (ECR) ion source and charge-bred to $q=28^{+}$. It was then accelerated through the ATLAS radio-frequency quadrupole (RFQ) and linac to $659 \mathrm{MeV}$ and focused onto a $1.1 \mathrm{mg} / \mathrm{cm}^{2}{ }^{208} \mathrm{~Pb}$ target (99.86\% enriched). The average ${ }^{146} \mathrm{Ba}$ beam intensity was $3 \times 10^{3}$ ions per second over 12 days. There were additional stable contaminants (having the same $A / q$ ) introduced into the beam from the ECR source, including ${ }^{94} \mathrm{Mo}^{18+},{ }^{94} \mathrm{Zr}^{18+},{ }^{120} \mathrm{Sn}^{23+},{ }^{193} \mathrm{Ir}^{37+}$, and ${ }^{198} \mathrm{Hg}^{38+}$, however all of them were easily separated from the $A=146$ beam ions based on time-of-flight (TOF) and scattering angle $(\theta)$ data recorded in $\mathrm{CHICO} 2$ (Fig. 1). This allowed for a clean $A=146 \gamma$-ray spectrum in GRETINA recorded from the Coulomb-excited beam ions (Fig. 2). 


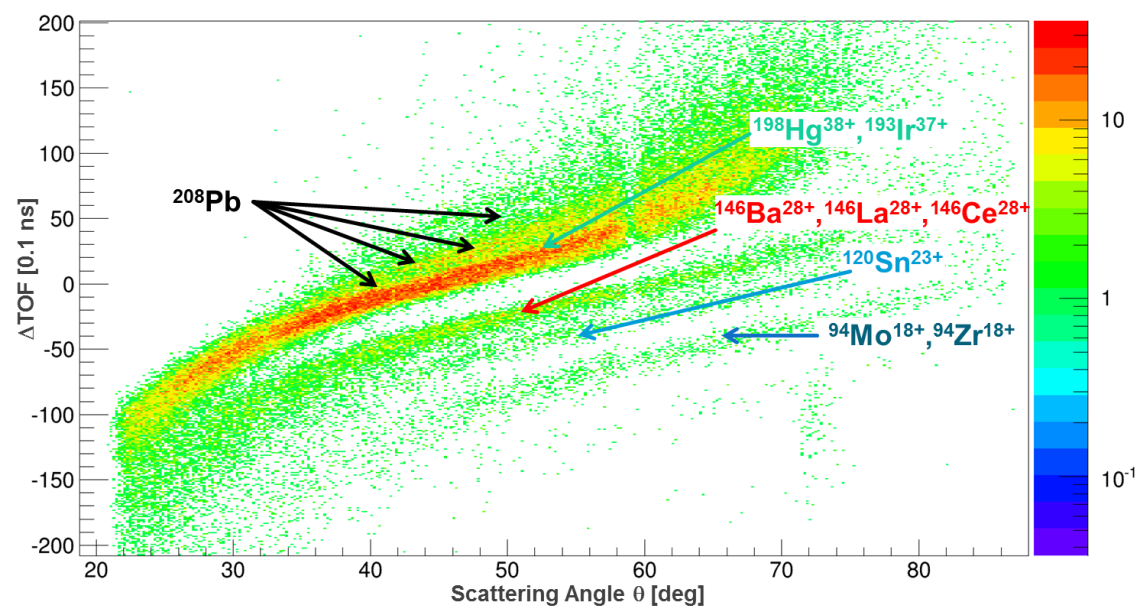

Figure 1: TOF vs. scattering angle recorded by $\mathrm{CHICO} 2$ in coincidence with a $\gamma$ ray recorded in GRETINA. The $A=146$ group is easily distinguished from the stable beam contaminants.

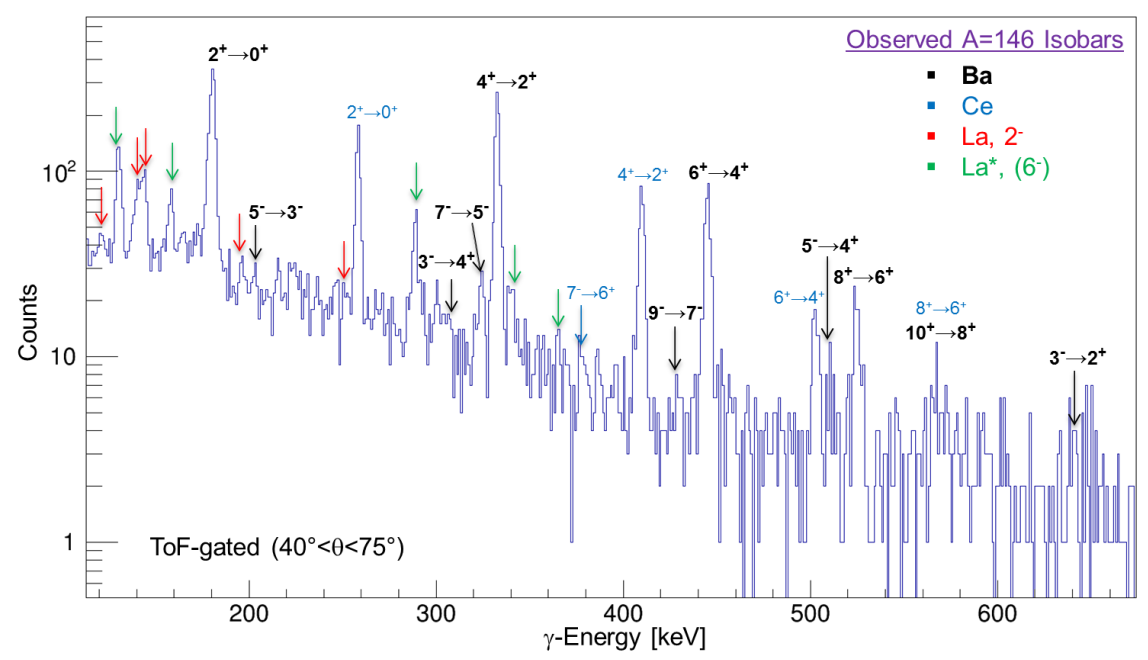

Figure 2: The coincident $\gamma$-ray spectrum by gating on the $A=146$ group in the CHICO2 TOF spectrum (Fig. 1). Many ${ }^{146} \mathrm{Ba}$ lines are seen along with lines from the radioactive isobaric contaminants that were also produced in CARIBU.

\section{Analysis}

In the $\gamma$-ray spectrum of Fig. 2, several peaks from ${ }^{146} \mathrm{Ba}$ are apparent, especially ones belonging to positive-parity levels in the ground-state band which are excited (and decay) by E2 transitions. The negative-parity levels are excited much less frequently, but the excitation occurs predominantly through $E 3$ transitions and their decay yields provide a measurement of the corresponding $E 3$ matrix elements.

The decay yields were extracted for two separate scattering angle ranges: $30^{\circ}-40^{\circ}$ and $40^{\circ}-$ $75^{\circ}$. At lower angles, it is difficult to isolate the $A=146$ ions from the other beam contaminants in 
the TOF spectrum, while at higher angles there is simply not enough statistics for a useful analysis as the cross section falls off with the well-known Rutherford angular dependence $1 / \sin ^{4}\left(\frac{\theta}{2}\right)$. The yields for each set of angles were analyzed using the Coulomb excitation least-squares search code, GOSIA $[13,14]$. Decay yields were observed for levels up to $10 \hbar$ in the ground-state band and $9 \hbar$ in the negative-parity band. The energies of all observed $\gamma$ rays, along with several branching ratios and lifetimes, were known in advance based on previous experiments $[12,15,16]$. The latter information was useful for constraining the GOSIA fit. The sets of $E 1, E 2$, and $E 3$ matrix elements between levels with no previously known lifetimes, however, were coupled according to the rigid-rotor prescription [17] governed by the individual intrinsic $E \lambda$ moments (see also Refs. [4, $6,18]$ ). This was done to help search for the $\chi^{2}$ minimum while excluding minima in unphysical regions of the parameter space which often arise from matrix elements that are relatively insensitive to the observed yields and other available experimental data (so-called nuisance parameters, see Ref. [14]). Once the $\chi^{2}$ minimum was found, the rigid-rotor constraint was removed to properly determine the full uncertainties, including correlations between matrix elements [19].

\section{Results and Discussion}

The final results from the GOSIA analysis for the $E 1$ matrix elements did not display much sensitivity to the experimental data. This is not surprising since the $E 1$ strength is known to be relatively weak [15] with the only observed $E 1$ decays coming from the $3^{-}$and $5^{-}$levels. Moreover, the relative sign between the intrinsic $E 1$ and $E 3$ moments was found to be completely insensitive to the data. On the other hand, a number of new $E 2$ and $E 3$ matrix elements were well-determined from the new Coulomb excitation yields; the full set of which are reported in Ref. [19]. The most significant result is the ground-state $E 3$ matrix element $\left\langle 3_{1}^{-}\|\mathscr{M}(E 3)\| 0_{1}^{+}\right\rangle$determined to be $0.65\left({ }_{-20}^{+14}\right) e b^{3 / 2}$ which reflects the amplitude of octupole deformation present in the ground state [2]. This corresponds to a $B\left(E 3 ; 3^{-} \rightarrow 0^{+}\right)$strength of $48\left({ }_{-29}^{+21}\right)$ W.u. which is very close to the value recently reported [6] for ${ }^{144} \mathrm{Ba}$ (Fig. 3).

The new measurement lends strong support to the idea that octupole collectivity is indeed enhanced in these barium isotopes near $N=90$, even exceeding the magnitude predicted in past beyond-mean-field calculations [21, 22]. This is also apparent when compared to Coulomb excitation measurements performed on the stable barium isotopes [20], where no octupole enhancement is expected to come from the valence neutrons. The observed enhancement at $N=90$ is also consistent with the interpretation that the strong octupole correlations are driven by the presence of the high- $j$, unique-parity $v i_{13 / 2}$ orbital near the Fermi surface (Fig. 4) and its interaction with a nearlyfilled $v f_{7 / 2}$ orbital [23]. Indeed, recent symmetry-conserving configuration-mixing (SCCM) calculations have provided quantitative support of this interpretation [24, 19].

\section{Conclusions}

With the present measurement, the low-energy octupole strength in ${ }^{146} \mathrm{Ba}$ has been quantified. Such a measurement was only made possible by recent advances in radioactive beam acceleration (CARIBU) and state-of-the-art detector arrays (GRETINA and CHICO2). The results confirm past theoretical predictions of enhanced $E 3$ transitions in this nucleus and are consistent with an 


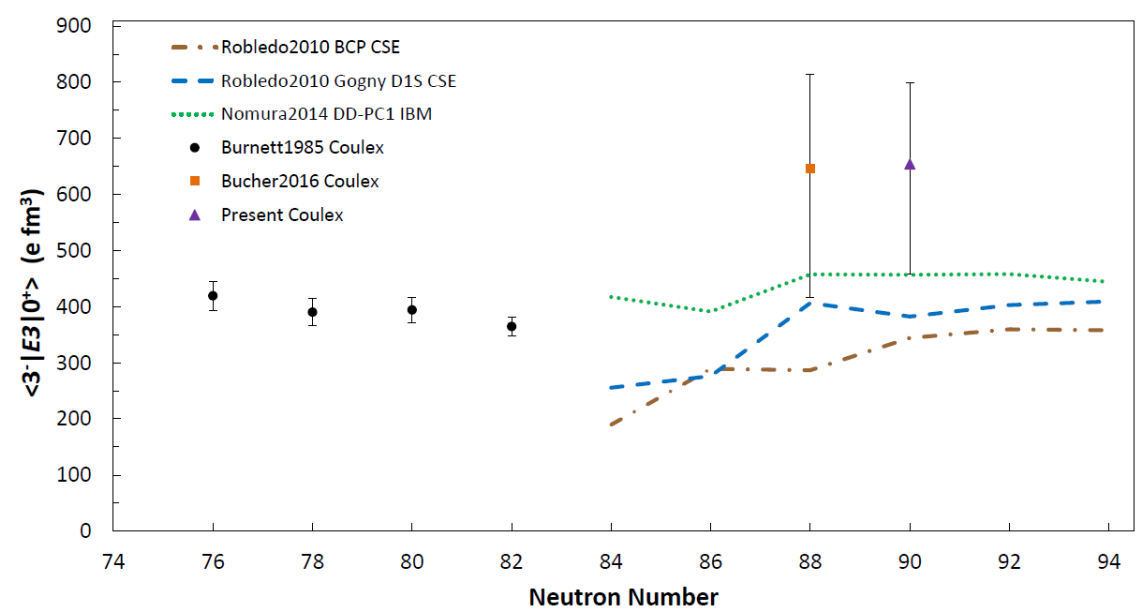

Figure 3: Values of the ground-state $E 3$ matrix elements for neutron-rich barium $(Z=56)$ isotopes are shown based on experimental work by Burnett et al. [20] and Bucher et al. [6] as well as theoretical work by Robledo et al. [21] and Nomura et al. [22].

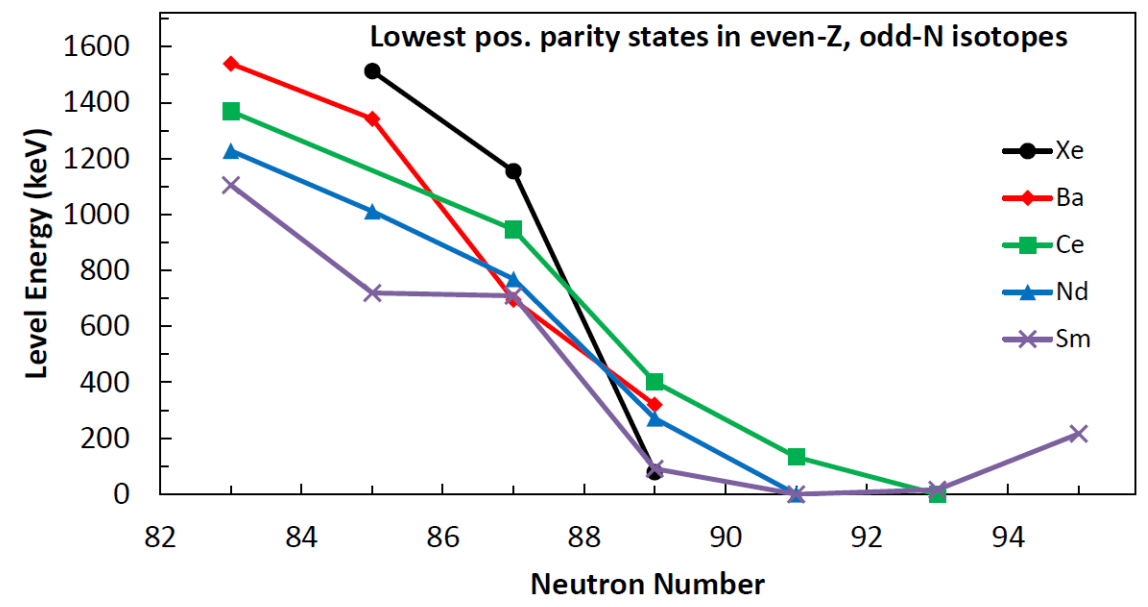

Figure 4: Energies of the first-excited positive-parity states in nearby even- $Z$, odd- $N$ isotopes. These lowenergy states must originate from the unique-parity spherical $v i_{13 / 2}$ single-particle orbitals. Clearly they approach the Fermi surface as neutrons are added towards $N=90$. (Data taken from the NNDC nuclear database [25].)

octupole-deformed ground state, similar to observations in neighboring ${ }^{144} \mathrm{Ba}$. The strong octupole correlations exhibited by these neutron-rich barium isotopes can be linked to the presence of $v i_{13 / 2^{-}}$ originating orbitals near the Fermi surface at $N=90$ in conjuction with a nearly-full set of $f_{7 / 2}$ valence neutrons populated there. As a result, this region of isotopes surrounding ${ }^{146} \mathrm{Ba}$, other than the neutron-deficient actinides around ${ }^{226} \mathrm{Ra}$, may represent the most suitable combinations of protons and neutrons for nuclei to form stable octupole-deformed shapes. 


\section{Acknowledgments}

This material is based upon work supported by the U.S. Department of Energy, Office of Science, Office of Nuclear Physics under Contract No. DE-AC02-06CH11357 (ANL). Work at LLNL and INL is supported by the U.S. DOE under respective Contracts No. DE-AC52-07NA27344 and No. DE-AC07-05ID14517. GRETINA was funded by the U.S. DOE-Office of Science, Office of Nuclear Physics by the ANL contract No. above and by Contract No. DE-AC02-05CH11231 (LBNL). This research used resources of ANL's ATLAS facility, which is a DOE Office of Science User Facility.

\section{References}

[1] F. S. Stephens, F. Asaro and I. Perlman, Radiations from 1- States in Even-Even Nuclei, Phys. Rev. 100 (1543) 1955

[2] P. A. Butler and W. Nazarewicz, Intrinsic Reflection Asymmetry in Atomic Nuclei, Rev. Mod. Phys. 68 (349) 1996

[3] R. H. Spear and W. N. Catford, Information on Octupole Deformation from the Systematics of $B\left(E 3 ; 0_{1}^{+} \rightarrow 3_{1}^{-}\right)$Values, Phys. Rev. C 41 (R1351) 1990

[4] H. J. Wollersheim et al., Coulomb Excitation of ${ }^{226}$ Ra, Nucl. Phys. A 556 (261) 1993

[5] L. P. Gaffney et al., Studies of Pear-Shaped Nuclei Using Accelerated Radioactive Beams, Nature (London) 497 (199) 2013

[6] B. Bucher et al., Direct Evidence of Octupole Deformation in Neutron-Rich ${ }^{144}$ Ba, Phys. Rev. Lett. 116 (112503) 2016

[7] G. Savard, S. Baker, C. Davids, A. F. Levand, E. F. Moore, R. C. Pardo, R. Vondrasek, B. Zabransky and G. Zinkann, Radioactive Beams from Gas Catchers: The CARIBU Facility, Nucl. Instrum. Methods B 266 (4086) 2008

[8] G. Savard, A. Levand, R. Pardo, R. Vondrasek and B. Zabransky, The CARIBU Facility, JPS Conf. Proc. 6 (010008) 2015

[9] D. Voulot, F. Wenander, E. Piselli, R. Scrivens, M. Lindroos, H. B. Jeppesen, L. M. Fraile, S. Sturm and P. Delahaye, Radioactive Beams at REX-ISOLDE: Present Status and Latest Developments, Nucl. Instrum. Methods B 266 (4103) 2008

[10] C. Y. Wu, D. Cline, A. Hayes, R. S. Flight, A. M. Melchionna, C. Zhou, I. Y. Lee, D. Swan, R. Fox and J. T. Anderson, CHICO2, A Two-Dimensional Pixelated Parallel-Plate Avalanche Counter, Nucl. Instrum. Methods A 814 (6) 2016

[11] S. Paschalis et al., The Performance of the Gamma-Ray Energy Tracking In-beam Nuclear Array GRETINA, Nucl. Instrum. Methods A 709 (44) 2013

[12] L. K. Peker and J. K. Tuli, Nuclear Data Sheets for A=146, Nucl. Data Sheets 82 (187) 1997

[13] T. Czosnyka, D. Cline and C. Y. Wu, Bull. Am. Phys. Soc. 28 (745) 1983

[14] D. Cline, T. Czosnyka, A. B. Hayes, P. Napiorkowski, N. Warr and C. Y. Wu, GOSIA User Manual for Simulation and Analysis of Coulomb Excitation Experiments, http://www.pas.rochester.edu/ cline/Gosia/Gosia_Manual_20120510.pdf, accessed March 2014 
[15] W. Urban et al., Octupole Correlations in Neutron-Rich, Even-Even Barium Isotopes, Nucl. Phys. A 613 (107) 1997

[16] H. Mach, W. Nazarewicz, D. Kusnezov, M. Moszyński, B. Fogelberg, M. Hellstrom, L. Spanier, R. L. Gill, R. F. Casten and A. Wolf, Influence of Shell Effects and Stable Octupole Deformation on the E1 and E2 Transition Rates in the Heavy-Ba Region, Phys. Rev. C 41 (R2469) 1990

[17] A. Bohr and B. R. Mottelson, Nuclear Structure Vol. 2, W. A. Benjamin, Reading, MA 1975

[18] W. R. Phillips, I. Ahmad, H. Emling, R. Holzmann, R. V. F. Janssens, T.-L. Khoo and M. W. Drigert, Octupole Deformation in Neutron-Rich Barium Isotopes, Phys. Rev. Lett. 57 (3257) 1986

[19] B. Bucher et al., Direct Evidence for Octupole Deformation in ${ }^{146}$ Ba and the Origin of Large E1 Moment Variations in Reflection-Asymmetric Nuclei, submitted to Phys. Rev. Lett. 2017

[20] S. M. Burnett, A. M. Baxter, S. Hinds, F. Pribac, R. H. Spear and W. J. Vermeer, A Measurement of $B\left(E 3 ; 0^{+} \rightarrow 3_{1}^{-}\right)$and Some E2 Transition Probabilities in ${ }^{132,134,136,138}$ Ba Using Coulomb Excitation, Nucl. Phys. A 432 (514) 1985

[21] L. M. Robledo, M. Baldo, P. Schuck and X. Viñas, Octupole Deformation Properties of the Barcelona-Catania-Paris Energy Density Functionals, Phys. Rev. C 81 (034315) 2010

[22] K. Nomura, D. Vretenar, T. Nikšić and B.-N. Lu, Microscopic Description of Octupole Shape-Phase Transitions in Light Actinide and Rare-Earth Nuclei, Phys. Rev. C 89 (024312) 2014

[23] I. Ahmad and P. A. Butler, Octupole Shapes in Nuclei, Annu. Rev. Nucl. Part. Sci. 43 (71) 1993

[24] T. R. Rodríguez and L. M. Robledo, private communication

[25] Brookhaven National Laboratory, NNDC Nuclear Database, http://www.nndc.bnl.gov, accessed May 2016 